\title{
Sébastien Roux
}

Chargé de recherche au Cnrs, LISST-Cas (UMR 5193)

Avec le support du programme ANR ETHOPOL ANR-14-CE29-0002

\section{L'Etat des origines \\ Histoires adoptives, conflits biographiques et vérités passées}

\section{Résumé}

La «question des origines » traverse les débats contemporains sur la filiation; il faudrait aujourd'hui, pour la sécurité psychique des enfants, révéler les conditions initiales des conceptions et/ou les existences antérieures à leur arrivée au sein des familles. En se concentrant sur le cas de l'adoption internationale, cette contribution interroge les effets sociaux et politiques de cette nouvelle injonction à la «transparence ", et questionne les modalités pratiques qui permettent l'édiction d'une histoire. Mettre en mots " son » passé et y « accéder » sont autant d'actions individuelles pour partie conditionnées par la force des Etats et leur politique d'archivage, révélant l'épaisseur public des récits (auto-)biographiques. 


\section{L'ETAT DES ORIGINES HISTOIRES ADOPTIVES, CONFLITS BIOGRAPHIQUES ET VERITES PASSEES}

Mai 2013. Aujourd'hui, j'accompagne Laure, une assistante sociale du service adoption du département de la Sauldre ${ }^{\mathrm{i}}$. Nous rendons visite aux Flamant. M. et Mme Flamant ont adopté il y a 3 ans un "petit Bulgare ». Né Mihayl, il a été renommé Mickaël à son arrivée en France. Il est aujourd'hui âgé de 7 ans ; c'est un petit garçon aux cheveux noirs et à la peau mate, dynamique et expansif. Les Flamant nous reçoivent dans leur grande maison individuelle dans la périphérie de Renangeours - la préfecture du département de l'ouest de la France dans lequel j'ai conduit l'un des volets français de mon enquête. M. Flamant travaille comme ingénieur dans l'aéronautique; son épouse est juriste dans une entreprise d'outillage industriel. Nous passons près d'une heure ensemble, à la grande satisfaction de Laure qui m'avait prévenu avant notre rencontre avoir « un petit coup de cœur pour ce couple et cette famille ».

Les premiers moments passés ensemble sont l'occasion de recueillir d'excellentes nouvelles concernant l'enfant. D'après les Flamant, Mickaël s'intègre parfaitement à l'école, est doué pour se faire des amis, aime le sport, la musique et jouit d'une très bonne santé. Tout en rappelant de temps à autre « qu'il faut parfois faire attention, parce que les adoptés peuvent être dans la surenchère pour plaire à leurs nouveaux parents ", Laure semble convaincue de la description qu'on nous propose. Après une trentaine de minutes, elle se tourne vers moi et m'explique, en présence des Flamant. «Tu sais, ils ont vraiment su comment expliquer à Mickaël d'où il venait ». Puis, s'adressant au couple : «Vous avez la boîte, et tout ? Mickaël peut toujours y accéder quand il veut? » Ils sourient, et saisissent un petit coffre en bois posé au sol, sous la table basse. Ils l'ouvrent. À l'intérieur, je découvre des photos de Mickaël, un petit lapin en peluche usé, quelques lettres. Tous ce artefacts ont été ramenés de l'institution bulgare dans laquelle l'enfant a grandi. Laura me regarde et acquiesce : "Tu vois, ça, c'est exactement ce qu'il faut faire $»$.

Cette scène dessine les contours du consensus (Lenoir 1986) actuellement en vigueur dans le monde de l'adoption et, plus largement, autour des "nouvelles" configurations familiales. D'après l'écrasante majorité des discours actuels sur l'enfance, notamment lorsque la filiation ne s'inscrit pas dans la naturalité supposée de la reproduction conjugale hétérosexuelle, il faudrait toujours déployer un ensemble de mesures, de pratiques et de discours afin de faciliter la reconstitution d'un récit biographique unifié permettant aux enfants, sans restriction d'âge ou de condition, d'accéder à «la vérité » de leur histoire. Ce récit serait même aujourd'hui devenu une nécessité, permettant d'assurer - par le verbe et la «transparence »- les conditions de l'épanouissement. Ainsi, et toujours pour le bien de l'enfant, il faudrait savoir mettre en mots l'histoire de « la dame qui t'a porté » (GPA), de « ta première maman » (accouchement sous $\mathrm{X}$ ) ou du «monsieur qui nous a aidé » (don de sperme).

Cette croyance contemporaine quant à la nécessité quasi anthropologique d'être capable d'accéder « au récit de ses origines »-ou, du moins, d'en formuler une version - se déploie dans le monde de l'adoption, notamment internationale. Les différences phénotypiques ont conduit à nommer et identifier, dans la plupart des cas ${ }^{\mathrm{ii}}$, « le pays où tu es né ». Cette mise en mots s'est opposée aux pratiques antérieures (réelles ou mythifiées) qui 
auraient dominé les décennies précédentes. À l'encontre d'un régime du secret et du silence qui aurait prévalu jusqu'aux années 1970, on favorise désormais une parole sur le passé pour la sécurité psychique actuelle et future des enfants. Et un nouvel impératif se formalise ; il faudrait aujourd'hui dire, rappeler, raviver, entretenir un lien entre le présent et l'histoire, pour que l'enfant sache quelle fut « sa vie d'avant ».

Cet article ne vise pas à prendre le contre-pied du consensus actuel sur les « origines » et leur accès, au risque de contredire sans arguments l'expérience des professionnel-le-s du soin psychique ou de délégitimer certaines revendications militantes. Certes, la défense d'un droit à l'accès à ses origines soulève un certain nombre d'interrogations, notamment dans une perspective féministe - en effet, un accès facilité à ces informations peut avoir des conséquences directes dans le traitement d'autres populations, au premier rang desquels les géniteurs - et plus particulièrement les génitrices. Mais il s'agit moins ici de s'opposer à l'argument de la nécessité ou de l'utilité d'un droit à l'accès aux origines personnelles, que d'interroger ce que dit ce besoin (plus historique qu'anthropologique) et, à travers lui, questionner ce que révèlent - et produisent - les pratiques et les discours qui entourent ce traitement du passé. La «question des origines" invite en effet à s'interroger sur l'appartenance des sujets, leur passé, leur mémoire et, plus largement, les formes contemporaines d'édiction d'une vérité biographique. Or la plupart des réflexions sur les origines traitent cet enjeu comme une question privée, gérée au plan individuel dans l'intimité des familles (avec le soutien des professionnel-le-s). Dans cette contribution, je souhaite montrer que la question des origines ne touche pas que les individus; elle interroge aussi les Etats, confrontés à des enjeux de traçabilité (Memmi 2014) et des exigences d'enregistrement qui interrogent la manière d'appréhender le pouvoir et ses effets dans la construction des subjectivités contemporaines.

\section{Méthodologie}

Cette réflexion sur les origines, leur sens politique et leur gestion publique s'inscrit dans un projet de long terme sur l'adoption internationale, conduit depuis $2013^{\mathrm{iii}}$. Plusieurs séquences d'enquête ethnographique ont été menées dans une pluralité d'espaces liés à l'adoption internationale. J'ai ainsi enquêté 5 mois en immersion dans une unité de l'Aide sociale à l'enfance en 2013, pour observer la prise en charge institutionnelle des parents-candidats et le suivi pré, per et post-adoption. J'ai également assisté à plusieurs journées de consultations dans des unités pédiatriques hospitalières spécialisées dans le conseil médical (COCA), en 2014, afin de mieux saisir la manière dont les parents-candidats appréhendent les pathologies possibles de leurs futurs enfants. Enfin, j'ai mené des observations et des entretiens en Ethiopie à l'occasion de deux séjours d'une durée totale de 3 mois réalisés en 2015 et 2016.

Ces terrains doivent être complétés par d'autres, en cours de négociation. Cette enquête multi-située (Marcus, 1995) vise à éclairer des facettes complémentaires de la question adoptive, chaque lieu d'enquête s'organisant autour d'une problématique spécifique (morale, santé, encadrement, etc.) 


\section{Un monde d'origines}

Dans le $1^{\mathrm{er}}$ tome de l'Histoire de la sexualité - La Volonté de savoir (1976), Michel Foucault montre que le régime répressif dans lequel nous pensons vivre est en réalité un monde bavard sur le sexe et les inquiétudes qu'il suscite. Or le traitement des origines s'apparente aujourd'hui à celui de la sexualité. Comme elle, la question des origines est au cœur d'intenses débats scientifiques, juridiques, médiatiques, politiques; elle fait l'objet d'expertises, de politiques publiques et de prises de position; elle sature les pratiques et les discours des professionnels de l'enfance et/ou de la reproduction. Pourtant, nous la pensons dissimulée, cachée, ou empêchée. Alors même que la plupart de nos contemporains appellent à «faciliter » leur accès, à "lever le voile » sur les conditions de la naissance, ou à "mettre enfin un terme au secret », n'est-on pas plutôt dans un moment ou la parole sur le passé est toujours déjà omniprésente ? Où "dire », " expliciter », " parler », "mettre en mots ", « rappeler», " encourager» sont des actions qui visent, continuellement, à formuler et reformuler un récit de soi qui valorise la connaissance des agencements qui ont présidé à la vérité de «sa » conception, de «son » enfance et de «son » histoire ?

Cette omniprésence a des conséquences directes sur la manière dont les sujets se pensent, comme sur la façon dont ils sont saisis par les institutions qui les encadrent. En m'intéressant successivement à deux niveaux d'intervention - l'un, plus microsociologique, sur les actions induites par le discours sur les origines, l'autre, plus macrosociologique, sur les dispositifs internationaux qui définissent les politiques d'archivages - je montrerai comment la prégnance et la transversalité d'un souci biographique transforme la manière d'appréhender les appartenances et les identités.

\section{Panser, suturer, unifier}

Ce matin, le Dr. Michel Varin reçoit dans une Consultation d'Orientation et de Conseils en Adoption (COCA) du sud de la France. Ce pédiatre d'une soixantaine d'années accueille M. et Mme Gentaly, accompagnés de leurs deux enfants - Louve, 8 mois, et Antoine, 4 ans. Les Gentaly sont désemparés. Antoine est exagérément agressif avec ses parents, les défie constamment et part dans des crises de hurlement qui épuisent sa famille. Le Dr. Varin prend rapidement connaissance du problème ; à aucun moment il n'est fait mention explicitement des conditions de l'adoption ni de la vie pré-adoptive d'Antoine ni de sa sœur. Dès que $M$. et Mme Gentaly terminent d'exposer la situation, le médecin se tourne vers le petit garçon. Il hausse la voix et, d'un ton bourru, entame un long monologue :

« Antoine, tu n'as pas à être méchant avec ta maman et ton papa. Ecoutemoi bien. Tu avais une autre maman avant, mais elle n'a pas pu te garder. Des fois, ce n'est pas possible de rester avec ses parents de naissance. Mais elle t'aimait beaucoup. Elle tenait beaucoup à toi. Si tu n'es plus avec elle, ce n'est pas parce qu'elle ne t'aimait pas. Mais aujourd'hui tu es avec ton papa et ta maman de France. [Le garçon se détourne ostensiblement et enfonce sa tête dans la poitrine de sa mère]. Oh, tu ne veux pas m'entendre, mais tu m'entends très bien. Je sais que tu m'entends. Et il faut que tu écoutes. [Antoine se bouche les oreilles et commence à gémir] Ce sont des 
choses très importantes que je te dis là. Tu avais une première maman, et aujourd'hui tu as une seconde maman, et un second papa. Et ta maman d'avant, elle t'aimait, et ta maman d'aujourd'hui elle t'aime aussi. Et tu n'as pas à être méchante avec elle. »

Le discours se poursuit et se répète pendant une dizaine de minutes, toujours sur le même thème. M. et Mme Gentaly semblent gênés. Le garçon s'est désormais calmé, mais continue de tourner le dos au pédiatre. Dès que le Dr. Varin termine de parler, Antoine hurle qu'il veut partir. Le médecin se tourne vers les parents et les sermonne en leur expliquant qu'ils doivent d'eux-mêmes rappeler à l'enfant ses origines, susciter la parole si elle ne vient pas, et rassurer leur fils sur la continuité d'un amour parental qui - paradoxalement s'exercerait indépendamment des parents. Enfin, il les encourage à prendre rapidement contact avec des associations d'adoptés afin de mener des activités avec d'autres familles adoptives, et «continuer surtout à parler de l'histoire d'Antoine ». M. et Mme Gentaly ânonnent, se lèvent, remercient et prennent congé.

$\mathrm{Au}$-delà du cas particulier et des réserves que cette scène peut susciter, la situation traduit la prégnance du discours sur les origines et explique pour partie la force de son succès. En effet, rappeler - voire produire - une biographie antérieure à «l'arrivée de l'enfant » est aujourd'hui pensé comme une nécessité, un devoir que l'on attend et exige des parents. Lors de sa consultation, Michel Varin a moins traité la violence et l'agressivité d'Antoine qu'il ne les a saisis comme le symptôme d'une incompétence parentale, voire d'une inaptitude. La fabrique des liens adoptifs - et, plus généralement, familiaux - ne saurait se dispenser aujourd'hui d'un encadrement prolongé où experts psycho-technocrates (Howell, 2006a) travailleurs sociaux, psychologues, pédiatres, etc. - «accompagnent » (et contrôlent) les parents dans leur apprentissage de la parentalité (Roux et Vozari, 2017). Dans le cadre de l'adoption internationale, cet apprentissage implique la verbalisation constante de «l'avant »; c'est par le rappel continu de son passé pré-adoptif que l'enfant doit saisir la linéarité de son existence. Son abandon, son déplacement et son arrivée au sein de sa famille adoptive étant systématiquement perçus comme traumatiques - et ce, quelles que soient les raisons de son adoption, son âge et son parcours - la mise en mots réitérée du passé permet de créer le sentiment d'une continuité, toujours déjà envisagée comme apaisante et rassurante.

Ainsi, la possibilité de formuler le récit de ses origines est aujourd'hui perçue comme une nécessité psychologique, et les réformes juridiques qui doivent la faciliter un impératif humaniste. Cette perspective est aujourd'hui largement dominante parmi les professionnels de l'adoption et de la filiation (services d'aide sociale à l'enfance, psychologues, travailleurs sociaux, etc.) $)^{\text {iv }}$ Dans le monde de l'adoption, ce consensus s'assoit sur une clinique psychologique et psychiatrique qui affirme le malaise - actuel ou latent - des adopté-e-s auxquelles on aurait caché (consciemment ou non) des vérités passées. Il est également renforcé par la visibilité d'un mouvement associatif qui relaie, amplifie et diffuse l'incompréhension et la colère d'enfants devenus adultes n'ayant pas eu accès aux informations les concernant préalablement à leur adoption. En France, « la Voix des adoptés » (ou VDA) est aujourd'hui le mouvement le plus visible et le plus relayé ; il se mobilise pour une transformation de la législation (notamment de l'adoption plénière) et une verbalisation accrue du vécu pré-adoptif.

Pourtant, si l'accès aux origines est aujourd'hui envisagé au sein du monde adoptif comme une question individuelle et psychologique, il est aussi en enjeu public. En effet, le traitement des origines recoupe directement des pratiques d'Etat, et invite à questionner la manière dont l'identité est produite par des dispositifs juridiques et administratifs spécifiques. Dans le contexte actuel, l'Etat n'est souvent appréhendé que comme une administration à 
réformer afin de reconnaître à chacun la possibilité de savoir « d'où » il/elle vient. Pourtant, il est aussi l'opérateur qui conditionne la possibilité même d'établir le récit quant à son passé, par la collecte et le stockage d'informations bureaucratiques standardisées (état-civil, registres, identification, etc.) Sans s'y réduire, la connaissance de «son » passé est donc toujours déjà liée aux traces qu'archive la puissance publique. D'autre part, la mémoire que l'Etat entretient vis-à-vis de ses sujets est aussi la marque de son pouvoir, et le produit d'une longue histoire d'identification des populations où la force publique dit qui (et que) sont celles et ceux qu'elle gouverne (Genèses, 2004 ; Noiriel, 2010). Ainsi, penser les origines invite à resituer les interrogations individuelles sur son passé et sa biographie dans un contexte plus large où ces questionnements, s'ils touchent à la singularité des sujets et au «sentiment de soi » (Fine, 2008), n'en sont pas moins des affaires d'Etat.

\section{Une éthique d'état-civil}

Le traitement actuel des origines pour se saisir à l'intersection de deux dynamiques, articulées mais spécifiques : la filiation et l'identification. Connaître ses origines, c'est à la fois s'inscrire dans une lignée et se positionner dans sa parenté, mais c'est aussi être désigné, enregistré et reconnu par un dispositif légitime visant à une "réduction sociale de l'incertitude sur les personnes » (Lignier, 2015, p. 180). En effet, accéder à ses origines, ce n'est pas qu'un « besoin » personnel ; c'est aussi adhérer à un dispositif qui pense nécessaire l'énonciation d'une vérité des sujets - pour eux-mêmes comme pour autrui. Les interrogations sur les origines se placent à l'intersection de deux processus, sans jamais se réduire à un seul. Elles sont autant le produit d'une volonté d'apaisement des souffrances psychiques causées par une méconnaissance de soi, qu'elles s'inscrivent dans une «morale d'Etat-civil » qui dit officiellement "ce que l'on est et demanderait de rester le même » (Foucault, 1969). Les inquiétudes autour des origines s'apparentent ainsi à un processus qui entend énoncer une vérité de soi suffisamment définitive pour contenter et apaiser à la fois le sujet et son Etat, à une éthique qui vise - dans le même temps - au bien individuel et à la satisfaction d'un intérêt public.

Si l'histoire des controverses récentes autour de l'accès aux origines personnelles a déjà été retracé (Ensellem, 2004 ; Séraphin, 2009), quelques rappels historiques permettent d'en saisir les formes actuelles. La possibilité d'accéder à ses origines s'est d'abord formulée comme la revendication d'un droit. Initialement portée par certains enfants "nés sous X » (devenus adultes) et/ou par des enfants adoptés sous le régime de l'adoption plénière, il visait à mettre un terme à l'anonymat garanti par le régime juridique français depuis les années 1950 et 1960 (1953 et 1959 pour l'accouchement sous X, 1966 pour l'adoption plénière) ${ }^{\mathrm{V}}$. En effet, dans ces deux situations, l'identité de la génitrice (accouchement sous X) ou du/des parent(s) de naissance (adoption plénière) n'était pas conservée, l'enfant étant - pour l'Etat sans parent biologique et/ou né de ses parents adoptifs. Ces dispositions se sont vues attaquées par comme parentalo-centrées à partir des années 1990. Pour ces opposants, souvent d'anciens adoptés épaulés par des psychologues et psychanalystes, l'impossibilité d'accéder à la «vérité de leurs histoires » résultait d'un dispositif juridique favorisant le droit des adultes sur celui des enfants.

Dans ce contexte particulier, la levée de l'anonymat remporte progressivement son combat; depuis la fin des années 1990, de nouvelles dispositions juridiques sont adoptées, effritant toujours davantage la possibilité du secret. Le 22 janvier 2002 Ségolène Royal, alors Ministre de la famille, transforme la gestion administrative de l'accouchement sous X et créé le Conseil national pour l'accès aux origines personnelles (Cnaop) ${ }^{\mathrm{vi}}$. Il est désormais possible 
- voire souhaitable - qu'une femme accouchant dans l'anonymat laisse des informations auxquelles l'enfant, devenu adulte, pourra accéder s'il/elle le souhaite. L'Etat n'est plus que le garant d'un anonymat protecteur; il " protège » aussi dorénavant les enfants en déployant un dispositif assurant une «traçabilité » (Memmi, 2014) partielle de ses sujets. Pour satisfaire le besoin et le désir de connaissance biographique, la filiation biologique devient rémanence ${ }^{\text {vii }}$. Si le lien entre la génitrice et celui qui devient aussi «son » enfant n'est pas indélébile (le nom de la génitrice peut toujours être tu, les informations la concernant n'étant pas nécessairement identificatoires), l'Etat organise toutefois le maintien d'une présence potentielle par une politique de collecte et d'archivage spécifique.

Cette dynamique touche aujourd'hui l'ensemble des questions relatives à la filiation, dans un contexte reproductif bouleversé par l'apparition des Nouvelles Technologies de Reproductions (NRT) (Strathern, 1992 ; Porqueres i Gené, 2009 ; Courduriès et Herbrand, 2014). FIV, dons de gamètes, d'œufs, PMA, voire GPA, autant de manières d'engendrer qui bousculent la croyance - voire le mythe - quant à l'évidence des liens familiaux. Ces « nouvelles » manières de faire famille s'adossent à des dispositifs bio-technologiques qui soulèvent des inquiétudes quant à l'affiliation des enfants. Face à la mise en circulation des gamètes, la diversification des techniques et la perte d'un sentiment d'évidence, les « origines » apparaissent comme un moyen privilégié d'accéder à la vérité des personnes. Dès lors, le secret devient un nouvel « intolérable » (Fassin et Bourdelais, 2005) et les dispositions initialement prévues pour protéger se transforment en violences à combattre. On comprend ainsi pourquoi la défense des origines revêt aujourd'hui les habits d'une démarche progressiste, à forte connotation juridique. D'une part, le débat s'est organisé comme la conquête d'un « droit» individuel mené au nom des enfants. D'autre part, les revendications sont aujourd'hui adressées à l'Etat, appelé à transformer ses pratiques et ses lois pour s'ajuster à une demande de re/connaissance, toujours déjà perçue comme légitime puisque curative, voire nécessaire ${ }^{\text {viii }}$.

Ces questionnements actuels sur la filiation et l'inscription des personnes dans leur parentèle recoupent ainsi des dynamiques plus anciennes sur l'édiction publique de la vérité biographique. Ils ne sont pas qu'une inquiétude relative au trouble que causerait une mise en question récente des liens familiaux, et notamment de la filiation. Ils sont aussi le produit d'une exigence publique d'identification où le pouvoir cherche à conserver, classer et organiser les informations sur les sujets qu'il gouverne. Cette logique d'identification, ancienne (About et Denis, 2010), s'est notamment traduite en France par l'instauration de l'état civil à la Révolution et la mise en place d'une administration spécialisée pour assurer notamment face à l'Eglise et ses registres (Noiriel, 1993) - une « surveillance des identités » (About, 2012) centralisée, adossée à une technologie de contrôle spécifique. Dès lors, penser « la question des origines » invite à articuler inquiétudes personnelles et logiques d'Etat, non en les opposant, mais en montrant comment elles s'unissent. S'affilier, c'est s'identifier; identifier, c'est affilier. Et si les initiatives publiques autour du « dévoilement » des origines tentent de «faire le bien » en répondant pour partie aux demandes de vérité qui leur sont adressées, ce bien recoupe aussi un intérêt public : celui de renforcer une capacité à diriger, par une politique éthique, des populations toujours davantage suivies, enregistrées et archivées.

\section{Géo/morale de l'adoption}

Dans le monde de l'adoption internationale cette exigence de «traçabilité des sujets » (à la fois individuelle et publique) est devenue depuis une vingtaine d'années un critère de 
moralité. Le dispositif qui organise ces circulations internationales d'enfants (Leinaweaver, 2008) est défini par un corps assez restreint de dispositions publiques internationales, définissant les conditions d'une adoption éthique. Le traité structurant demeure la Convention de la Haye sur la protection des enfants et la coopération en matière d'adoption internationale, signée le 29 mai 1993, dans le prolongement de la Convention internationale des Droits de l'enfant (1989) - acmé de l'activité juridique autour de la protection de l'enfance qui marque les années 1980 et $1990^{\text {ix }}$. Après des débuts difficiles, la Convention de 1993 est aujourd'hui ratifiée par plus de 90 pays, dont la plupart des principaux pays dits «d'accueil» et «d'origine ».

Parmi les nombreux points avancés par la Convention de La Haye, trois aspects méritent une attention particulière. Premièrement, la Convention hiérarchise la désirabilité relative des espaces éducatifs et pose un principe de subsidiarité pour déterminer «l'appartenance » des enfants. Les arguments préalables affirment en effet que « chaque Etat devrait prendre, par priorité, des mesures appropriées pour permettre le maintien dans sa famille d'origine », avant de préciser que «l'adoption internationale peut présenter l'avantage de donner une famille permanente à l'enfant pour lequel une famille appropriée ne peut être trouvée dans son État d'origine ». Ainsi l'architecture de la protection de l'enfance organise l'adoption à l'image de cercles concentriques. Au cœur, l'idéal domestique, affectif et pédagogique : le(s) parent(s) de naissance. Puis vient la famille de «sang » (oncles, tantes, grands-parents, cousins, etc.). Ensuite, une famille adoptive du même Etat peut être recherchée. Enfin, l'adoption internationale peut être envisagée si, et seulement si, l'enfant n'est pas adopté dans son Etat de naissance. Dès lors, tout se passe comme si la recherche de "l'intérêt supérieur de l'enfant» superposait implicitement bonheur et proximité ; et la Convention de La Haye affirme comme principes le bénéfice affectif et la supériorité morale du proche sur le lointain, du génétique sur le social et du national sur l'étranger.

Ensuite, et ce point est lié au précédent, les Etats membres de la Convention sont invités à conduire une politique d'archivage spécifique. L'article 30, notamment, précise :

«Les autorités compétentes d'un Etat contractant veillent à conserver les informations qu'elles détiennent sur les origines de l'enfant, notamment celles relatives à l'identité de sa mère et de son père, ainsi que les données sur le passé médical de l'enfant et de sa famille. Elles assurent l'accès de l'enfant ou de son représentant à ces informations, avec les conseils appropriés, dans la mesure permise par la loi de leur Etat $»$.

Certes, la dernière incise de l'article rappelle la possibilité, pour les Etats membres, d'une législation particulière pour encadrer «l'accès » aux informations dont ils disposent. Mais il n'en demeure pas moins que la Convention impose, comme pratique éthique, une politique où les informations biographiques des enfants et de leurs familles successives (de naissance et d'adoption) sont gérées par une politique d'archivage spécifique. Or, non seulement cette disposition maintient un lien entre l'enfant et son espace d'origine, mais elle confie également à la puissance publique un devoir moral: celui de conserver, indépendamment de la citoyenneté acquise par l'enfant et de sa destination, des données standardisées sur sa condition originelle.

La Convention de la Haye est aujourd'hui célébrée comme l'outil juridique le plus efficace pour garantir le respect de «standards éthiques ». Le poids de la Convention de la Haye apparaît aujourd'hui d'autant plus nécessaire que le monde de l'adoption internationale traverse une crise sans précédent. En effet, le nombre d'enfants adoptés à l'international a décru d'environ $70 \%$ depuis le début des années 2000, en France comme à l'étranger. Pour le 
sociologue et démographe Jean-François Mignot, « ce n'est pas la 'demande' des couples ou des individus candidats à l'adoption qui a baissé, mais bien 'l'offre' de mineurs adoptables » (2015, p. 3). L'auteur explique cette tendance par la conjonction d'une multitude de facteurs : des « raisons structurelles, démographiques ou économiques (...) dans les pays d'origine » (augmentation du niveau de vie, diffusion de la contraception et de l'IVG, etc.), ainsi que des «décisions de nature politique et juridique» dans les Etats habituellement pourvoyeurs d'enfants (comme la Chine ou la Russie). J-F Mignot évoque également l'influence de la Convention de la Haye et rappelle qu'un certain nombre de pays ont mis en place des moratoires visant à aligner leurs politiques et leurs pratiques sur les nouvelles recommandations en vigueur. En effet, la Convention est aujourd'hui d'autant plus incontournable qu'elle est la cause et la solution de la crise qu'elle engendre. Le texte est pour partie responsable d'une diminution des circulations d'enfants (Roux, 2015) - augmentant les délais d'attente dans les pays d'accueil, transformant voire empêchant la réalisation de la majorité des projets parentaux, diminuant les revenus des orphelinats dans les pays d'origine, surchargeant les institutions locales, encombrant les tribunaux, etc. Mais il est aussi perçu comme le garant d'une adoption "propre », dont tous - administrations, parents, enfants, professionnels, etc. - sont invités à se réjouir, pour aujourd'hui comme pour l'avenir.

\section{Les ambiguïtés de l'avant}

Dans ce contexte particulier, où « la moralisation » de l'adoption internationale affirme des principes universalisés qui, à leur tour, façonnent de nouvelles pratiques bureaucratiques et renforcent le pouvoir des Etats, j'ai choisi de conduire une part de mon enquête ethnographique en Ethiopie. L'Ethiopie se classe depuis une quinzaine d'années parmi les principaux pays d'origine pour les adoptants Français-es. Le nombre de personnes adoptées et l'histoire longue de l'adoption dans ce pays se traduisent par une visibilité croissante des discours d'enfants qui - devenus adultes - reviennent sur les conditions de leurs adoptions. Or, le cas éthiopien est particulièrement discuté. Dix, quinze, vingt ans après les faits, certains témoignent de situations hautement problématiques, contraires aux standards actuels d'une adoption "éthique»: des parents seraient toujours vivants, des documents auraient été falsifiés, des intermédiaires se seraient enrichis, des histoires auraient été inventées... Certains adoptés procèdent aujourd'hui à des retours plus ou moins longs sur la trace de leur passé et cherchent, entre doutes et espoirs, à renouer avec une histoire dont on les aurait privés. D'autre part, l'Ethiopie n'est toujours pas officiellement signataire de la Convention de La Haye. Cette politique nationale avive les inquiétudes : celles des parents adoptants, en France et à l'étranger, qui craignent que l'enfant qu'on leur propose n'ait pas été recueilli dans des conditions «éthiques"; celles des professionnels de l'adoption, qui découragent de plus en plus les candidats à cette destination et/ou s'interrogent sur les adoptions déjà réalisées ; celles des diplomaties étrangères, certains gouvernements européens ayant suspendu les nouvelles procédures suite à des suspicions de malversations criminelles... Autant de craintes qui permettent de saisir l'importance et l'influence des nouvelles normes éthiques en vigueur dans le monde de l'adoption internationale et la place qu'occupent aujourd'hui les Etats dans l'édiction des identités. 
En mars 2016, je rencontre Samira (Lucie) ${ }^{\mathrm{x}}$ à une terrasse d'un hôtel d'Addis. C'est une jeune femme de 24 ans, menue, aux cheveux longs et crépus, à la peau foncée. Elle est tendue, un peu inquiète. Mais elle «a accepté de parler parce qu'[elle] veut dire des choses ». Notre entretien dure un peu plus d'une heure et demie, durant lesquelles la jeune femme enchaîne les macchiatos et les cigarettes. Au fur et à mesure de nos échanges, les réticences initiales laissent place à la colère, au ressentiment, parfois aux pleurs. Samira est née en Ethiopie en 1991. En 1999, les Meunier l'adoptent avec son petit frère, Dawit (Thomas). Samira et Dawit sont la troisième adoption du couple. Leur mère, Michelle, est avocate ; leur père, Jean, haut fonctionnaire dans l'administration hospitalière. Le couple est infertile. Catholiques pratiquants, ils se sont tourné vers l'adoption internationale dès le début des années 1990 pour fonder la famille nombreuse dont ils rêvaient alors. Une fois obtenu leur agrément - l'autorisation administrative d'adopter (Villeneuve-Gokalp, 2007) - les Meunier se tournent vers un intermédiaire habilité : une Organisation Autorisée pour l'Adoption (ou OAA) $)^{\mathrm{xi}}$, que j'appellerai ici les Enfants De La Lumière - ou ED2L. Cette association religieuse les oriente initialement vers le Vietnam où elle dispose d'une antenne facilitant les démarches avec les autorités locales. Grâce aux ED2L, les Meunier adoptent en 1991 Luc (Hoan), âgé de 8 mois lorsqu'il arrive en France. En 1993, deux ans plus tard et toujours avec les ED2L, les Meunier adoptent Paul, cette fois-ci originaire d'Haïti ; il arrive un peu plus âgé (5 ans). Enfin, en 1999, c'est au tour de Lucie (Samira) et Thomas (Dawit), venus d'Ethiopie.

Avant d'être adoptée, Samira a passé deux ans avec Dawit dans un orphelinat d'Addis Abeba, Le Refuge, où elle a été transférée avec son petit frère quelques semaines après avoir été recueillie dans le Nord du pays, à Ruki, à une quinzaine d'heures de route de la capitale, au cœur des hauts plateaux. Elle arrive au Refuge à l'âge de 6 ans; elle en partira à 8. Samira se souvient d'années difficiles, de quelques maltraitances aussi même si elle tient à préciser « qu'elle n'a jamais eu faim ». Un jour, après une longue attente où ils voient la plupart de leurs ami-e-s partir pour l'Europe et les Etats-Unis, la directrice vient voir les deux enfants. C'est leur tour; on leur a trouvé des parents. Samira se rappelle qu'on la lave, la coiffe, l'apprête; des photos sont envoyées. L'attente dure 2 mois. À l'époque, le droit éthiopien n'impose pas la présence des adoptants. Les OAA envoient des «cigognes », ces membres bénévoles - le plus souvent d'anciens parents adoptifs investis dans l'association - qui repartent en avion accompagnés d'une quinzaine d'enfants. Samira se souvient de son départ :

« On est parti de l'aéroport de nuit. On a fait une escale en Italie ; pas trop de souvenirs. Mais quand on est arrivé à Roissy, pendant $48 \mathrm{~h} . .$. je n'ai eu aucun sentiment. Rien. C'est le seul moment de ma vie où je ne ressens ni tristesse, ni peine, rien. Le néant. J'ai bien compris que l'Ethiopie, c'était fini. À l'aéroport, il y a toutes les familles qui nous accueillent. [...] Tout le monde vient, les grands-parents, les cousins, les parents, les frères et sœurs... Je n'ai rien compris [...] On arrive avec tout le monde, dans le hall. On rencontre notre famille dans le hall [silence]. »

Les mois passent, puis les années. Samira s'adapte à la France, à son village, à sa nouvelle vie. Puis, vers l'âge de 12 ans, elle commence à interroger ses parents. Elle prétend qu'elle a une famille restée à Ruki. Des frères et des sœurs. Ses parents doutent : d'après le dossier que leur a fourni les ED2L, Dawit et elle sont «des enfants des rues aux parents décédés sans famille identifiée ». Mais Samira insiste. Elle sait bien que ses parents de naissance sont morts ; elle se rappelle même des circonstances. Ils ont été emportés par un accident de bus lorsqu'elle avait 5 ans. Mais elle se souvient aussi d'une fratrie nombreuse et se rappelle désormais du jour où son oncle l'a abandonnée à Ruki. Elle dit y avoir été 
recueillie «par une dame» qui a organisé son transfert au Refuge. Michelle et Jean la questionnent : est-ce qu'elle ne confondrait pas d'autres pensionnaires de l'orphelinat d'Addis avec des «frères et sœurs » de Ruki ? Est-ce que des traumas d'enfance troubleraient ses souvenirs ? Le dossier de l'orphelinat est bien formel : «enfants des rues aux parents décédés sans famille identifiée $»$.

Samira vit une adolescence difficile. Elle affronte ses parents adoptifs, explique qu'elle «n'a rien à faire là », que «sa vraie famille l'attend»; ses résultats scolaires plongent. Elle devient violente envers les autres, et aussi parfois envers elle-même. Avec le soutien de ses parents français, Samira «commence des démarches ». Mais elle se heurte à un mur :

« J'ai écrit à la dame des ED2L. Plutôt sur le mode "qu'est-ce que je fais là ? Il faut que quelqu'un me réponde. J'ai ma famille là-bas. Qu'est-ce qu'il se passe ?" [...] Jusqu'à mes 18 ans, j'ai écrit des lettres à des médecins ou des gens qui travaillaient au Refuge. Pas d'informations, pas de retour positif. Juste : “Ce n'est pas possible", “ce n'est pas possible”. [...] On me disait : "Non, on n'a rien". [...] Juste : "On n'a rien". "

Quelques jours après sa majorité, Samira décide de réaliser le projet qui l'anime depuis des années. Elle achète un billet d'avion avec l'argent d'un job d'été et décide de se rendre directement à l'orphelinat. Elle part à Addis «avec un ami qui était dans la même démarche qu'[elle], pour faire la recherche ». La jeune femme se présente au Refuge, armée de son album-photo d'enfance et d'une ferme résolution. Le dossier officiel de l'orphelinat précise bien que Samira et Dawit sont des enfants des rues recueillis à Ruki avant d'être transférés à Addis. Le jugement d'adoption rédigé par la justice éthiopienne confirme cette version. Pourtant, sur place, Samira rencontre une employée, Beza, qui la prend à l'écart et lui propose de discuter à l'extérieur de l'orphelinat. À quelques encablures de l'institution, Beza lui annonce tout d'un coup que «sa famille la recherche ». Elle lui apprend qu'un homme s'est signalé il y a quelques mois au Refuge à la recherche d'enfants qu'il aurait déposé à Ruki plus de 10 ans auparavant.

«C'était l'oncle qui m'avait placé. J'étais restée 15 jours [à Ruki] avant d'être envoyée au Refuge. Cet oncle-là, ils l'avaient eu au téléphone... Je rigolais, je pleurais, je ne savais pas vraiment comment réagir, parce que c'était l'aboutissement de toute une vie [rires]. Après, je suis allée dans le bureau de la directrice du Refuge; une femme qui parle français. Elle m'a demandé si je voulais parler à mes sœurs. On les a appelés. Je ne parlais pas du tout l'amharique, c'est elle qui faisait la traduction. Ma grande sœur, quand elle a eu le coup de téléphone, elle est tombée dans les pommes ».

L'émotion est toujours présente lorsque Samira raconte ces retrouvailles. Elle reconnaît avoir " eu de la chance », « il y en a plein qui font le retour aussi mais qui, eux, ne retrouvent personne ». Samira m'explique ce qu'elle apprendra par la suite. Un an après la mort des parents de Samira et Dawit, son oncle avait décidé de placer les enfants pour soulager les frères et sœurs aînés qui, âgés d'une quinzaine d'années pour les plus grands, avaient commencé à travailler pour subvenir aux besoins des plus jeunes. Cet oncle aurait pris seul l'initiative, et aurait signé un document officiel attestant que les enfants avaient été trouvés abandonnés. Après quelques mois, et notamment face à la colère des frères et sœurs de Samira et Dawit, il aurait tenté de récupérer les enfants, sans succès. Les petits étaient déjà 
partis pour Addis. Après de longues recherches, la famille de Samira aurait contacté Le Refuge. L'orphelinat n'aurait pas donné suite. Il se serait d'abord retranché derrière les documents qui désignaient les enfants comme abandonnés, ensuite - une fois l'adoption prononcée - derrière le jugement officiel confiant Samira et Dawit aux Meunier. Mais la famille éthiopienne de Samira n'avait pas complètement perdu espoir; ils auraient tenté à plusieurs reprises de recontacter Le Refuge et d'autres institutions d'Addis, cherchant pendant des années la trace de leur frère et sœur disparus.

Quelques semaines avant l'arrivée de Samira en Ethiopie, sachant que la jeune femme allait fêter son dix-huitième anniversaire, ils avaient tenté une nouvelle fois de recontacter l'orphelinat. Le temps, mais aussi la multiplication des situations problématiques, les mises en cause récurrentes du Refuge et les attaques contre un système judiciaire accusé de corruption et/ou d'incompétence avaient conduit l'orphelinat à revoir sa politique de "confidentialité ». L'institution avait alors accepté «d'engager des démarches» pour réunifier les familles lorsque les versions semblaient suffisamment coïncider pour mettre en doute les papiers officiels. Samira, avec l'aide de Beza, reçoit ainsi la confirmation de ce qu'elle clamait depuis des années : elle a trois sœurs et deux frères en Ethiopie, tous vivants, tous plus âgés et qui, eux aussi, tentaient depuis près d'une décennie de réunir une famille paradoxalement disloquée par l'adoption. Depuis ce premier voyage, Samira est retournée à trois reprises «dans [s] on pays », dont une fois pour une période de 8 mois consécutifs ; aujourd'hui, elle dit vouloir s'installer définitivement à Addis Abeba. La jeune femme se dit apaisée, et m'explique à quel point elle se "sent mieux ", moins " déchirée » par le traumatisme d'une migration non voulue, et par la violence du mensonge biographique qu'elle a dû dévoiler.

Le cas de Samira est exceptionnel ; pas sa situation. J'ai été régulièrement confronté à des récits similaires. Bien sûr, tous les enfants «d'origine éthiopienne » n'ont pas dû traverser des expériences aussi difficiles, ni n'ont été confrontés à de tels mensonges biographiques. Mais la plupart des personnes adoptées que j'ai rencontrées, en France ou en Ethiopie, doutent de manière plus ou moins marquée des informations officielles qui les concernent. Le soupçon est omniprésent, et récurrent. Les communautés d'adoptés originaires d'Ethiopie, en France et à l'étranger, mettent en circulation des récits de "retours" où les versions officielles sont ébranlées; des sites internet se sont développés pour faciliter les « reconnexions », en mettant en relation des parents Ethiopiens avec des enfants adoptés; les réseaux sociaux sont de plus en plus mobilisés pour rétablir des liens familiaux disloqués; des structures privés se sont même montés pour enquêter, moyennant finances, sur l'histoire d'anciens adoptés qui doutent de leur situation et demandent, depuis l'Europe ou les EtatsUnis, une enquête privée.

Du côté éthiopien, et alors même que la durée de mon enquête était restreinte, j'ai pu rencontrer plusieurs femmes m'expliquant avoir abandonné leurs enfants et avoir fourni à l'administration, de manière plus ou moins éclairée, le récit nécessaire pour faciliter leurs départs. Les situations sont diverses : ces personnes ont pu présenter leurs propres enfants comme leurs neveux ou leurs nièces ; certaines affirment qu'elles n'avaient pas compris que les enfants ne reviendraient pas, et avaient compris les orphelinats comme des structures temporaires; pour d'autres, la pauvreté et la violence qu'elles subissaient alors rendaient désirables un futur européen ou américain pour leurs enfants, quel qu'en soit le prix à payer. Certes, les motivations n'ont pas toujours été qu'altruistes : envoyer un enfant à l'étranger, c'était aussi espérer qu'il ou elle ramène un jour un peu d'argent, ou offre à la manière d'une diaspora expatriée des opportunités interdites en Ethiopie. Ces malentendus (Chabloz, 2007) aux conséquences tragiques expliquent d'ailleurs que les retrouvailles, lorsqu'elles ont lieu, soient souvent difficiles. Que sont devenus ces autres qu'on croyait siens ? Et comment vivre 
des liens familiaux lorsque la «vérité » des relations ressurgit après des années de séparation douloureuse?

La situation de Samira révèle une situation paradoxale, souvent peu interrogée : dans leurs quêtes des origines, à quelles informations les enfants (devenus adultes) parviennentils? Les parents de Samira et Dawit, familiers du monde de l'adoption, insérés dans les collectifs et informés des exigences psychologiques actuelles ont bien « facilité » l'accès de leurs enfants à «leurs origines »; ils n'ont jamais menti sur l'Ethiopie. Au contraire, les Meunier encourageaient régulièrement leurs enfants à envoyer des présents au Refuge et à maintenir un lien avec l'orphelinat «d'où ils venaient ». Mais la formalisation d'un discours sur soi ne requiert pas que la suture entre un avant et un présent délimité par la migration et les frontières nationales. Le passé de Samira n'était pas qu' «éthiopien »; ses souvenirs d'enfance lui ont rappelé qu'elle existait aussi avant son existence d'orpheline, et qu'accéder à «ses origines » était un processus conditionné par la véracité et la légitimité de traces publiques (ici falsifiées). En France, le débat actuel sur les origines présuppose toujours nécessaire un double combat. Une pédagogie des parents d'abord, auxquels il faudrait enseigner la transparence contre des désirs - plus ou moins conscients - de secret; une transformation des politiques publiques ensuite, pour pouvoir accéder toujours davantage à la vérité de soi contre des intérêts d'Etat. Pourtant « accéder à ses origines », ce n'est pas que se voir rappeler une condition initiale d'orphelin étranger. C'est aussi, et peut-être surtout, un processus de fabrique discursive qui ne peut établir la vérité de soi qu'à la condition de l'Etat. C'est lui, et lui seul, qui peut aujourd'hui garantir la véracité des documents à partir desquels les individus sont appelés à reconstruire la linéarité de leurs existences. Les défaillances éthiopiennes, et ses conséquences tragiques, rappellent que les origines ne sont pas contre l'Etat ; c'est lui, au contraire, qui les rend possibles.

\section{« J'ai tout »}

En Ethiopie l'omniprésence du soupçon transfigure l'adoption. Tous les agents impliqués sont désormais forcés de se positionner face à une situation devenue intenable. En décembre 2015, je me rends dans un orphelinat d'Addis Abeba tenu par quelques religieuses, aidées de volontaires internationaux. L'établissement, sobre, est propre et bien rangé, malgré l'odeur écœurante d'urine et de lait aigre caractéristique des orphelinats éthiopiens. Au début des années 1990, La Miséricorde du Sacré Cour a collaboré avec Le Refuge avant de prendre ses distances avec l'institution. Sœur Lazare, la directrice, me reçoit ; elle avait accepté par email de me rencontrer pour me présenter son travail et ses activités. Affable lors de nos premières minutes de conversation, elle se montre rapidement inquiète lorsqu'elle comprend que mes questions ne s'arrêteront pas à la version édulcorée que présente le site internet. Lorsque je parle du Refuge que j'ai visité quelques jours plus tôt, elle se tord les mains ; d'elle-même, et sans que je ne mentionne les enjeux éthiques autour de l'adoptabilité, elle m'explique, dans un débit soudain accéléré : «Moi les dossiers, je les ai. J'ai tout. C'est tout ici. La personne qui veut venir voir, elle vient, elle voit. J'ai tout ». Et Sœur Lazare d'ouvrir en s'agitant un placard rempli de pochettes nominatives. Au hasard, elle prend un dossier, me le tend, se ravise, le reprend et l'ouvre face à moi. «Lui, par exemple, vous voyez. Ce gamin, je sais qu'il a été adopté il y a 7 ans, de quelle province il vient, comment sont morts ses parents, les jugements légaux, le tribunal, tout $»$. Un peu surpris de sa réaction - j'étais alors encore peu familier de la récurrence des doutes quant à la provenance des enfants en Ethiopie... - je lui demande de préciser. Elle lève les bras au ciel et ajoute, un peu théâtrale : 
«Je n'ai rien à me reprocher, tout est légal. Légal. Pas de secrets. Pas de mensonges [No secrets. No Lies]. La Miséricorde a toujours été éthique. Les dossiers sont là. »

Sœur Lazare a raison : les documents sont légaux. Mais la légalité ne fait pas la vérité. L'Ethiopie est un pays régulièrement dénoncé pour la sévérité de son administration, la force de son pouvoir judiciaro-policier, et le degré élevé de surveillance de sa population. Lorsque Mengistu quitte le pouvoir en 1991, quelques années après la chute du Derg (1974-1987), il laisse aux mains du nouveau gouvernement dirigé par le FDRPE un Etat exsangue, mais assis sur les structures répressives des régimes antérieurs ${ }^{\text {xii }}$. La population est surveillée, enregistrée, réprimée. Pour autant, les documents sont aisément falsifiés. J'ai régulièrement tenté de rencontrer des responsables administratifs éthiopiens pour les interroger sur les incohérences des registres nationaux et l'importance des « retours » actuels d'anciens adoptés. Les rendez-vous, lorsqu'ils étaient accordés, étaient ensuite quasi systématiquement annulés. J'ai également reçu des mises en garde plus ou moins voilées et des «invitations » à réorienter ma recherche. En recueillant des récits d'abandon, et en recoupant des témoignages divers, il apparaît que les enfants étaient souvent mal enregistrés à leurs naissances, surtout dans les coins les plus reculés. Les registres, s'ils existent, sont peu fiables, surtout dans les régions rurales; les enfants ont souvent été déclarés plusieurs années après leur naissance, particulièrement dans les années 1990 lorsque le pays était secoué par la famine, la guerre (avec l'Erythrée) et une désorganisation massive de l'administration suite à la chute du Derg. Qui plus est, la corruption est endémique. D'après un diplomate de haut rang en charge des questions adoptives travaillant au sein d'un consulat européen :

« Tout le monde sait que la plupart des papiers sont faux, quand ils existent. Je passe mon temps à faire comme si je croyais que les informations éthiopiennes que je suis amené à traiter étaient vraies. Mais c'est faux. Je le sais. Les jugements sont faux. Les dates de naissance sont fausses. Les situations familiales sont fausses. L'Etat ment, la justice ment, les orphelinats mentent. Pas toujours, mais souvent. Il y a trop de corruption ici ».

Le format de mon enquête, initialement centrée sur les adoptions, les orphelinats et la fabrique des liens familiaux ne m'avait pas préparé à un tel degré de corruption, ni ne m'avait armé pour penser une situation bureaucratique où la surveillance et l'enregistrement des personnes est - paradoxalement - omniprésente mais incomplète. C'est en rencontrant des adoptés, en recueillant des récits de vie et en collectant des témoignages de personnes engagées dans le monde de l'adoption que j'ai progressivement saisi la puissance de l'Etat dans la fabrique des identités, à partir de ses manques. Le cas éthiopien a ainsi fonctionné comme un révélateur. Ses dysfonctionnements pointent ce que l'on n'interroge plus dans nos sociétés : les traces officielles que l'on cherche à re/collecter pour connaitre « son » histoire n'ont de valeur que lorsque l'Etat leur confère statut de vérité.

\section{Une fin}

Le 4 mai 2016, le Quai d'Orsay publie un communiqué officiel suspendant toute nouvelle procédure d'adoption en Ethiopie :

«La Mission de l'adoption internationale (MAI) informe les candidats à l'adoption en Éthiopie qu'en date du 22 avril 2016, le ministre 
des Affaires étrangères et du Développement international a adressé un courrier à Mme Zenebu Tadesse, ministre des Femmes et des Enfants en Éthiopie, annonçant la suspension des adoptions internationales jusqu'à la mise en place des réformes législatives et institutionnelles engagées par ce pays. D'autres pays d'accueil tels que l'Allemagne, la Belgique francophone, le Danemark l'Espagne, l'Irlande, la Suède et la Suisse ont déjà suspendu les adoptions en Éthiopie. Cette décision intervient à la suite de la mission de la MAI en Éthiopie effectuée du 10 au 12 février dernier. Lors de cette mission, la MAI a rencontré la ministre des Femmes et des Enfants pour faire un état des lieux sur la situation de l'adoption. Le constat a été fait conjointement de la nécessité de suspendre les adoptions internationales pour garantir l'éthique et la sécurité juridique des procédures et afin d'encourager les prises en charge locales alternatives à l'adoption internationale. »

Certes, les arrivées avaient déjà diminué. D'après les statistiques du Ministère des Affaires étrangères français, 220 enfants ont été adoptés dans ce pays en 2012, 140 en 2013, 52 en 2014 et seulement 24 en 2015. Mais, alors même que la demande d'enfants adoptés ne faiblit pas, il devient dorénavant impossible pour la France de poursuivre les adoptions en Ethiopie. Le pays est accusé, en termes diplomatiques, de manquements «éthiques »: il ne sait garantir la provenance de ses sujets.

Régulièrement, les Ethiopiens que j'ai rencontrés à Addis Abeba m'ont expliqué ne pas comprendre cette situation. Beaucoup s'en sont ouverts, avec une amertume et une défiance mêlées. Beza par exemple, l'employée du Refuge qui depuis quelques années voit son travail bouleversé par le nombre croissant d'anciens adoptés à la recherche de leur passé m'explique un soir, résumant en quelques mots une position partagée par beaucoup :

«Tous ceux qui veulent revenir, qui veulent retrouver leurs familles, ils disent que c'est ici chez eux... Et qu'ils sont malheureux. Mais c'est quoi leur malheur? Ils seraient restés ici, ils seraient pauvres, sans éducation, à vendre des billets de taxis collectifs pour 2 birrs ou à dormir dans la rue. A l'orphelinat, il y'a combien de gamins qui rêvent de partir? Maintenant, c'est plus possible. Ils vont devenir quoi, ces gosses ? Ces anciens adoptés, ce qu'ils appellent leur malheur, c'était leur chance. Qu'est-ce qu'ils viennent nous embêter avec leurs problèmes de psychologie? C'est vraiment des trucs de riches et de Blancs, parce que eux ils ont survécu et qu'ils ont oublié. »

Sans adhérer nécessairement à ce type de discours, leur récurrence exprime une hiérarchie du malheur que l'on retrouve fréquemment à Addis, et qui explique aussi, pour partie, les incompréhensions qui traversent ces situations. "Partir» a longtemps été perçu comme l'objectif qui justifiait le mensonge. Une fin, au-delà des moyens ; une opportunité aussi. Pour des enfants, mais aussi pour celles et ceux qui ont pu s'enrichir du commerce de l'adoption (intermédiaires, institutions, autorités locales certainement). Une chance enfin, pour les « orphelins » comme pour leurs nouveaux parents, à qui aurait été offert la possibilité d'une vie familiale idéale.

Pourtant la douleur de Samira et d'autres rappelle que le malheur ne se situe pas nécessairement où on le pense, ni où on l'anticipe. Les recommandations éthiques qui organisent aujourd'hui les circulations internationales d'enfants imposent une traçabilité des 
adoptés. Il faut désormais, pour apaiser par avance des souffrances à venir, déployer un dispositif de suivi transnational aligné sur des standards situés d'identification des sujets. L'adoption, plus qu'une nouvelle naissance, est aujourd'hui envisagée comme une étape biographique. Mais la continuité des vies et la possibilité pour les individus de se ressaisir (aujourd'hui ou après), dans une histoire linéaire, est un processus qui requiert plus qu'un récit. Il nécessite des marques, des traces et des archives auxquelles on confère un caractère indéniable, un Etat suffisamment fort et légitime pour que le gouvernement qu'il exerce sur ses sujets les aide à re/constituer leurs histoires. La question des « origines » montre ainsi, à partir d'une situation intime et personnelle, comment le pouvoir, s'il contrôle, enregistre et contraint, devient aussi - dans certaines situations - une condition au bonheur. Etre tracé, enregistré, surveillé permet aussi de se ressaisir et se penser comme sujet. La «bonne gestion » publique des individus rejoint pour partie leurs volontés individuelles. Et la marque des appartenances apparaît d'autant plus forte et nécessaire qu'elle réunit besoin individuel et souci d'Etat faisant de l'identification, plus qu'une surveillance, une action éthique.

\section{Bibliographie}

About, Ilsen. 2011. « Surveillance des identités et régime colonial en Indochine, 1890-1912», Criminocorpus [En ligne]

About, Ilsen \& Denis, Vincent. 2010. Histoire de l'identification des personnes, Paris, La Découverte.

Chabloz, Nadège. 2007. «Le malentendu. Les rencontres paradoxales du "tourisme solidaire" », Actes de la recherche en sciences sociales, $\mathrm{n}^{\circ} 170: 32-47$

Courduriès, Jérôme \& Cathy, Herbrand. 2014. «Genre, parenté et techniques de reproduction assistée : bilan ». Enfances familles générations, $\mathrm{n}^{\circ} 21$ : I-XXVII.

Denéchère, Yves. 2011. Des enfants venus de loin histoire de l'adoption internationale en France. Paris : Armand Colin.

Ensellem, Cécile. 2004. Naître sans mère? Accouchement sous X et filiation. Rennes : PUR. Eng, David. 2010. The Feeling of Kinship: Queer Liberalism and the Racialization of Intimacy. Durham : Duke University Press.

Fassin, Didier \& Bourdelais, Patrice (dir.). 2005. Les constructions de l'intolérable : Etudes d'anthropologie et d'histoire sur les frontières de l'espace moral. Paris : La Découverte.

Fine Agnès (dir.). 2008. États civils en questions. Papiers, identités, sentiment de soi. Paris : Éditions du CTHS.

Fine, Agnès \& Martial, Agnès. 2010. "Vers une naturalisation de la filiation? ", Genèses, $\mathrm{n}^{\circ} 78: 121-134$.

Foucault, Michel. 1976. Histoire de la sexualité, tome 1: La volonté de savoir. Paris : Gallimard.

Genèses, 2004. Dossier « Vos papiers! ", n 54.

Howell, Signe. 2006. « Changes in Moral Values About the Family. Adoption Legislation in Norway and the US », Social Analysis, n 50 (3) : 146-163.

Iacub, Marcela. 2004. L'empire du ventre. Pour une autre histoire de la maternité. Paris : Fayard.

Leinaweaver, Jessaca. 2008. The Circulation of Children: Kinship, Mobility, and Morality in Ayacucho. Durham : Duke University Press.

Lenoir, Rémi. 1986. "Groupes de pression et groupes consensuels. Contribution à une analyse de la formation du droit », Actes de la recherche en sciences sociales, ${ }^{\circ} 64: 30-39$. 
Lignier, Wilfried. 2015. «L'identification des enfants. Un modèle utile pour l'analyse des primes socialisations », Sociologie, vol. 6, $\mathrm{n}^{\circ} 2$ : 177-220.

Marcus, George. 1995. «Ethnography In/Of the World System: The Emergence of MultiSited Ethnography », Annual Review of Anthropology, n 24 : 95-117.

Marre, Diana \& Briggs, Laura. 2009. International Adoption: Global Inequalities and the Circulation of Children. New York : New York University Press.

Memmi, Dominique. 2014. La Revanche de la chair : Essai sur les nouveaux supports de l'identité. Paris : Le Seuil.

Mignot, Jean-François. 2015. «L'adoption internationale dans le monde: les raisons du déclin », Population \& Sociétés, n 519 , février.

Noiriel, Gérard. 1993. "L'identification des citoyens. Naissance de l'état-civil républicain », Genèses, $\mathrm{n}^{\circ} 13: 3-28$.

Noiriel, Gérard. 2007. L'identification. Genèse d'un travail d'État. Paris : Belin.

Porqueres i Gené, Enric (dir.). 2009. Défis contemporains de la parenté. Paris : Éditions de l'EHESS.

Roux, Sébastien. 2015. «La circulation internationale des enfants », in Trepeusch, Marie \& Steiner, Philippe (dir.), Les marchés contestés: Quand le marché rencontre la morale. Toulouse, PUM : 29-61.

Roux, Sébastien. 2017. « The Colour of Family Happiness. Adoption and the Racial Distribution of Children in Contemporary France », Social Anthropology/Anthropologie Sociale, sous presse.

Roux, Sébastien \& Vozari, Anne-Sophie. 2017. « Parents at their Best: The Ethopolitics of Family Bonding in France », Ethnography, sous presse.

Séraphin, Gilles. 2009. "L'accès aux origines : les ressorts d'un débat passionné », Esprit, $\mathrm{n}^{\circ} 5: 82-102$.

Strathern, Marilyn. 1992. Reproducing the Future: Anthropology, Kinship, and the New Reproductive Technologies, New York : Routledge.

Théry, Irène (dir.). 2014. Filiation, origines, parentalité. Le droit face aux nouvelles valeurs de responsabilité générationelle. Rapport au Ministère délégué chargé de la famille. Villeneuve-Gokalp, Catherine. 2004. «Du désir d'adoption à l'accueil d'un enfant. Une enquête en France », Population, n ${ }^{\circ} 67$ (2) : 281-314.

${ }^{\text {i }}$ Afin de préserver la confidentialité des informations recueillies, j'ai opté pour une stratégie d'anonymisation systématique. J'ai ainsi choisi de modifier les noms de personnes, de lieux et d'institutions quels que soient les espaces enquêtés. Seuls les noms des Etats (et leurs capitales) ont été conservés, les contextes nationaux déterminant les situations observées.

ii Sur la diversité phénotypique au sein des familles, et les processus de racialisation qui traversent l'adoption internationale, voir Eng (2010), Marre \& Briggs (2009) et Roux (2017). 
iii Cette recherche a bénéficié du soutien de l'ANR (projet ETHOPOL - ANR-14CE29-0002), ainsi que d'un appui financier du CESSP (UMR 8102), du LISST (UMR 5193) et d'un soutien logistique et scientifique du Centre Français des Etudes Ethiopiennes (CFEE, Addis Abeba). Je tiens également à remercier Jérôme Courduriès, Mélanie Gourarier, Sarah Mazouz, Massimo Prearo et les participant-e-s aux journées «Loyautés citoyennes » et «Transnational Politics» (qui se sont tenues en juin et septembre 2016 à la Humboldt Universität zu Berlin) pour la qualité de leurs remarques sur des versions préalables.

iv Il serait réducteur d'associer ce consensus à un-e auteur-e unique. On pourra toutefois utilement se référer aux écrits de la psychanalyste Geneviève Delaisi de Parseval et du juriste Pierre Verdier, tous deux engagés de longue date en faveur d'un « droit à l'accès à ses origines ", pour comprendre l'association opérée entre récit biographique, « transparence » et réponse à la souffrance des personnes dont les conditions de procréation, de naissance ou d'éducation auraient été passées sous silence.

${ }^{\vee}$ Je ne fais ici référence qu'aux décrets du 29 novembre 1953 et du 7 janvier 1959 (pour l'accouchement sous le secret) et à la loi du 11 juillet 1966 portant réforme de l'adoption (pour l'adoption plénière), car ces textes ont organisé l'architecture contemporaine de l'anonymat contre laquelle se sont mobilisés les partisans d'une réforme pour l'accès aux origines personnelles.

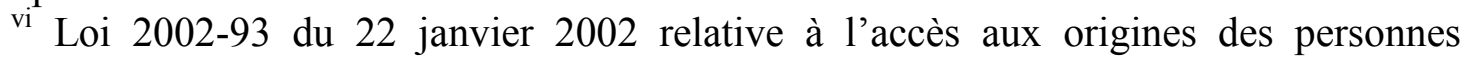
adoptées et pupilles de l'Etat.

${ }^{\text {vii }}$ Cet aspect a été critiqué avec virulence par Marcela Iacub, dans son essai L'empire $d u$ ventre (2004). Pour elle, la quête des origines - et notamment le combat que mènent certaines associations d'adoptés - peut s'interpréter comme la marque de leur attachement au biologique, et n'a donc pas à être satisfaite. Pour une mise en perspective de l'argument de la « nature » autour des questions de filiation, voir Fine et Martial (2010).

viii À cet égard, le rapport «Filiation, origines, parentalité » rendu au Ministère de la famille en 2014 et dirigé par Irène Théry est particulièrement représentatif (Théry, 2014). Sans me prononcer ici sur le bien-fondé ou non des revendications portées par ce groupe, il apparaît significatif que les auteurs aient accordé une place si prépondérante à la question des origines dans un écrit sur les réformes juridiques autour de la famille. Ils défendent en effet comme un progrès, pour le bien des enfants et de la société, des réformes administratives et juridiques facilitant la connaissance du passé et, à travers elle, la vérité des sujets.

${ }^{i x}$ La Convention des Nations Unies sur les Droits de l'enfant du 20 novembre 1989 demeure la principale disposition juridique internationale pour la protection de l'enfance. Si elle est souvent perçue comme le socle des dispositifs actuels, elle est aussi le produit d'une décennie de mobilisation pour l'universalisation des droits de l'enfant. Dans le domaine de l'adoption internationale, une autre Déclaration des Nations Unies - parfois oubliée - a posé les principes généraux repris par la Convention de 1989 et le Traité de 1993 : la Résolution de 1'Assemblée générale 41/85 du 3 décembre 1986. Je ne traiterai toutefois pas de ce texte dans le cadre de cet article, préférant me concentrer sur le Traité de 1993, qui demeure aujourd'hui le texte le plus mobilisé par les acteurs impliqués.

${ }^{\mathrm{x}}$ Dans la suite de l'article, j'emploierai systématiquement le prénom que les personnes choisissent d'utiliser pour se désigner. Parfois, comme ici, je préciserai le prénom officiellement enregistré à l'Etat-civil français.

${ }^{x i}$ En France, ces associations, habilitées au niveau départemental pour un ou plusieurs pays spécifiques, sont des intermédiaires bénévoles (mais facturant l'adoption au forfait) qui facilitent les démarches locales pour les adoptant-e-s. Héritières des « œuvres » (Denéchère 
Version auteur - Acceptée pour publication A paraître dans Genèses, nº 108 (La Reproduction nationale - 2017)

2011), ces associations étaient principalement d'obédience catholique humanitaire ; leur profil est aujourd'hui plus varié. Elles réalisent à l'heure actuelle la majorité des adoptions.

xii En 1974, le Derg remplace le régime impérial d'Haïlé Sélassié $1^{\mathrm{er}}$; Mengistu instaure une junte militaire d'inspiration marxiste et devient président de gouvernement militaire provisoire de l'Ethiopie socialiste (1977 - 1987). Il est chassé du pouvoir en 1991 par le Front Démocratique Révolutionnaire du Peuple Ethiopien (FDRPE), dirigé par Meles Zenawi (1955-2012) qui le remplace à la tête de l'Etat. En 1995, une nouvelle constitution proclame la République fédérale démocratique d'Ethiopie, constamment dirigée depuis sa création par le FDRPE. «Démocratie à parti unique » (les différents partis fédéraux étant des émanations du FDRPE), l'Ethiopie est régulièrement classée parmi les pays les plus répressifs dans la plupart des index mondiaux (Human Freedom Index, Index of Economic Freedom, Press Freedom Index, etc.) 